\section{The ethical challenges of in utero gene therapy}

In the past year, proposals to undertake in utero gene therapy have generated much discussion and debate. Concerns have been voiced that in utero gene therapy perhaps should never be undertaken $^{1}$. Although there are certainly reasons for caution with respect to the initiation of clinical trials of in utero gene therapy in humans, a persuasive case has not been made that such research is unethical.

There are many reasons for moving forward with in utero interventions. The most important is that for some diseases and disorders it makes sense to try to intervene as early as possible so as to prevent or slow dysfunction and morbidity. In addition, the developing fetus may be a better candidate for gene therapy than the adult. Stable and widespread gene engraftment may be more feasible in a fetus, where stem cells or pleuripotent progenitor cells are more accessible to vectors. Furthermore, the problem of immunologic responses to the vector and transgene product (that is, the therapeutic protein), which has plagued early attempts at post-natal somatic gene therapy, may be avoided in the fetus if the genetic intervention occurs during the period of immunologic development.

There are very real ethical concerns that must be addressed to undertake in utero gene therapy. Obviously, efforts at in utero gene therapy require thorough evaluation of safety and efficacy in animal models before contemplating clinical trials. Studies in animals have been promising, although more questions remain ${ }^{2}$. As any research in utero poses risks of infection, immune reactions and the induction of preterm labour, and poses risks for two parties, mother and fetus, the rationale for and methods of undertaking such work must meet the highest scientific and medical standards.

But those who have voiced concern about the ethics of in utero gene therapy have tended to focus on a different set of moral concerns. Much concern has been expressed about the possibility that in utero gene transfer will lead to the uptake and expression of genetic material in cells other then those that are the targets of gene therapy. Of particular concern is the possibility that genetic material may be incorporated into the germ line of subjects, leading to permanent changes that can be passed on to future individuals ${ }^{1,3}$.

The chances that the vector will access the germ cells and cause harmful effects are probably very low due to multiple physical and biological barriers. Ultimately these risks must be weighed against the risks of not intervening or the risk of a significant birth defect independent of gene therapy, which is estimated to occur in 1 of 50 live births ${ }^{4}$. This must be put into the context of risks that have already been accepted and incorporated into medical practice involving the fetus or the creation of embryos and fetuses.

The most likely candidates for in utero gene therapy are not likely to live to reproductive age. Even if they do, the chance of mating and producing a child in which incorporated genetic material causes disorder or disability is very small. If it has any effect, inadvertent change of germline materials through in utero gene transfer is likely to produce an individual incapable of producing offspring.

In our opinion, other concerns that have been expressed are even less persuasive then worries about inadvertent gene transfer. The fact that adverse changes may be difficult to detect is not an argu- ment against in utero gene therapy. It is merely an argument for closer monitoring of subjects. The fact that gene therapy has not yet proven effective in adults provides no basis for arguing at this stage in the evolution of gene therapy that research to establish efficacy in adults, children or fetuses should end. It merely reflects the fact that the technology is early in its development. Some families will not accept adoption, abortion or preimplantation diagnosis. For other conditions, no techniques exist for diagnosing fatal or severely disabling conditions. To argue that research on in utero gene therapy should not proceed when sufficient animal work has been done to establish safety and therapeutic promise ignores the moral claim that children with deleterious mutations or genetically based disorders and their families be able to seek treatments for these conditions.

The real moral challenge facing in utero gene therapy is to find ways to insure that the review of protocols is adequate, that those undertaking trials are competent to do so; that adequate financing exists to permit fair access to clinical trials; and that careful procedures are worked out for insuring informed consent, equity in subject selection and adequate oversight and review for the earliest clinical studies, in which the prospect of direct benefit to the fetus is tiny or non-existent. In our view, that is where the efforts of researchers, policy makers, regulators and ethicists ought to be directed.

Arthur L. Caplan ${ }^{1,2}$ \& James M. Wilson ${ }^{2}$ ${ }^{1}$ Center for Bioethics and ${ }^{2}$ Institute for Human Gene Therapy, University of Pennsylvania, Philadelphia, Pennsylvania, USA.

Correspondence should be addressed to J.M.W. (e-mail:wilsonjm@mail.med.upenn.edu).

1. Billings, P.R. Nature Med. 5, 255-256 (1999).

2. Schneider, H. \& Coutelle, C. Nature Med. 5, 256-257 (1999).

3. Clothier, C.M. Report of the Committee on the Ethics of Gene Therapy: presented to Parliament by command of Her Majesty, January 1992. p. 41 (London, Great Britain, 1992).

4. Emery, A.E.H. \& Rimoin, D.L. Nature and Incidence of Genetic Disease 1500 (Churchill Livingstone, Edinburgh, 1990). 